\title{
Lip-Reading Technique Using Spatio-Temporal Templates and Support Vector Machines
}

\author{
Wai Chee Yau, Dinesh Kant Kumar, and Tharangini Chinnadurai \\ School of Electrical and Computer Engineering, RMIT University \\ waichee@ieee.org
}

\begin{abstract}
This paper presents a lip-reading technique to identify the unspoken phones using support vector machines. The proposed system is based on temporal integration of the video data to generate spatiotemporal templates (STT). 64 Zernike moments (ZM) are extracted from each STT. This work proposes a novel feature selection algorithm to reduce the dimensionality of the $64 \mathrm{ZM}$ to 12 features. The proposed technique uses the shape of probability curve as a goodness measure for optimal feature selection. The feature vectors are classified using nonlinear support vector machines.Such a system could be invaluable when it is important to communicate without making a sound, such as giving passwords when in public spaces.
\end{abstract}

Keywords: visual speech recognition, motion segmentation, feature selection, Zernike moments, support vector machines.

\section{Introduction}

Speech recognition technologies provide a flexible and natural way for users to control computer through speech. Nevertheless, the performance of audio speech recognizers degrades when the sound signal strength is low, or in situations with high ambient noise levels. This research proposes to use video data related to mouth movements for lip-reading. Advantages of lip-reading system are : (i) not affected by audio noise (ii) not affected by change in acoustic conditions (iii) does not require the user to utter a sound.

Visual features proposed in the literature can be broadly categorized into shape-based, pixel-based and dynamic features. Shape-based features rely on the geometric shape of mouth or lips. The first lip-reading system was first developed by Petajan [1] using shape-based features such as height and width of the mouth. Researchers have reported on the use of artificial markers on the users mouth to extract lip contours 2. The use of artificial markers is not suitable for practical speech-controlled applications. Lip-reading systems that use pixel-based features assume that the pixel values around the mouth area contain salient speech information [3]. Other lip-reading methods using active contours to encode lip-shapes [5] and constrained lip-motion statistics [6] have also been reported in the literature. 
This paper proposes a lip-reading technique using spatio-temporal templates (STT). STT is created by temporal integration the video data and assigning greater weights to more recent movement. The resultant STT is a 2-D grayscale image that is suitable for representing short duration facial movement. This paper proposes a system where the camera is attached in place of the microphone to the commonly available headsets. Potamianos et. al. 4] have demonstrated that using mouth videos captured from cameras attached to wearable headset produced better results as compared to full face videos. Another advantage of this is that it is no longer required to identify the region of interest, reducing the computation required. The proposed visual speech recognition technique is divided into four phases: (i) motion segmentation, (ii) feature extraction and dimensionality reduction and (iii) classification.

\section{Motion Segmentation}

Mouth motion in each video is represented using a 2D grayscale image - spatiotemporal template (STT). The pixel intensity of a STT corresponds to a function of the temporal history of motion at that pixel location. The gray levels of a STT are the temporal descriptors of the motion [7. To minimize the effects of lighting conditions on the mouth motion representation ability of STT, a global illumination normalization method based on histogram equalization is applied on the images before computing STT.

Accumulative image differencing is applied on the image sequence by subtracting the intensity values between successive frames to generate a sequence of difference of frames (DOFs). DOF of the $t^{t h}$ frame obtained by subtracting consecutive frames. The delimiters for the start and stop of the motion are manually inserted into the image sequence of each utterance. The DOF are binarised using a fixed threshold value to generate a binary image, $B_{t}(x, y)$. An optimum, fixed threshold value is determined empirically. The intensity value of the STT at pixel location (x, y) of $t^{t h}$ frame is given by

$$
\operatorname{STT}_{t}(x, y)=\max \bigcup_{t=2}^{N} B_{t}(x, y) . t
$$

$N$ is the total number of frames used to capture the mouth motion. By computing the STT values for all the pixels coordinates $(\mathrm{x}, \mathrm{y})$ of the image sequence using Eq. 1 will produce a scalar-valued grayscale image (STT). STT of mouth video would have brighter pixels corresponding to more recent mouth movement and darker pixels corresponding to static elements.

The motivation of using STT in lip-reading is the ability of STT to remove static elements from the sequence of images and preserve the short duration mouth movement 8. STT is also invariant to the skin color of the speakers due to the image subtraction process. The proposed motion segmentation approach is computationally inexpensive and hence suitable for real time implementation. The speed of phonation of a speaker might vary for each repetition of a phoneme. 
This paper suggests a model to approximate such variations by normalizing the overall duration of an utterance. This is achieved by normalizing the intensity values of STT to be between 0 and 1.

\section{Feature Extraction}

This paper investigates the use of Zernike moments as image descriptors to represent STT. One of the motivations for using ZM is due to the simple rotational properties of ZM. ZM is one of the robust region-based shape descriptors stated in MPEG-7 standard 9. ZM is demonstrated to outperform other image moments such as geometric moments, Legendre moments and complex moments in terms of sensitivity to image noise, information redundancy and image representation capability [10.

$\mathrm{ZM}$ is computed by projecting image function $f(x, y)$ onto the orthogonal Zernike polynomial, $V_{n l}$. ZM is defined within a unit circle (i.e.: $x^{2}+y^{2} \leq 1$ ). $\mathrm{ZM}$ of order $n$ and repetition $l, Z_{n l}$ is given by

$$
Z_{n l}=\left[\frac{n+1}{\pi}\right] \int_{0}^{2 \pi} \int_{0}^{\infty}\left[V_{n l}(\rho, \theta)\right] f(\rho, \theta) d \rho d \theta
$$

$|l| \leq n$ and $(n-|l|)$ is even. $f(\rho, \theta)$ is the intensity distribution of STT mapped to a unit circle of radius $\rho$ and angle $\theta$ where $x=\rho \cos \theta$ and $y=\rho \sin \theta$. STT are scaled to be within a unit circle centered at the origin before computing ZM.

The rotation of the mouth in an image results in a phase shift on the ZM 11 and hence the absolute value of ZM is invariant to rotational changes. This paper uses the absolute value of ZM as features to represent STT. The optimal number of Zernike moments is determined empirically by additive method- starting with order 2 moments and increasing up to 81 moments. 64 moments is found to produce the best results and further increasing the number of moment did not improve the recognition rate. Hence 64 moments $\left(0^{\text {th }}\right.$ to $14^{\text {th }}$ order) were used in the experiments. The number of DCT features was kept the same as Zernike moments.64 Zernike moments (ZM) is computed from each STT of size $72 \times 72$ (a total of 5184 pixels).

\subsection{Feature Selection}

A well known recent algorithm that eliminates the redundant features and retains the features which are relevant with the class is Fast Correlation Based Filter algorithm (FCBF) [12] FCBF algorithm is faster, more accurate and results in less number of features than Principal Component Analysis (PCA) and Linear Discriminant Analysis (LDA) and this is confirmed in [13. However, the limitations of FCBF are:

- FCBF does not discard one of the two features, if they both have a consistent, predictable relationship between them, in all the instances for all the classes. 
- FCBF does not discard the features which are not uniquely related to each of the classes. This is an important factor to consider in feature optimisation because, even though a feature is very much relevant to the class, it is not an optimal feature, if it is not uniquely related to each of the classes.

A simple solution to identify both the above situations is to create a curve which contains the relative positions of the input instances, described as follows:

1. If the curve containing the relative positions are same (or a multiple of each other) for any two particular features (for all the classes), then we could discard one of those features, as one of them is a scaled copy of the other.

2. If the curve containing the relative positions are same (or a multiple of each other) for a particular feature in all the classes, then that feature could be discarded from the set of optimal features. This is because, the feature is no longer unique to the class.

The curve mentioned above is created from the input data (instances) by finding the relative position measure of a single value to the entire set of measurements. Percentiles offer one of the methods of comparing a single value with the complete set of measurements. The two important characteristics of this curve would be:

- The width of the curve, which indicates the range of the input values

- The skew of the curve, which indicates the median of the input values

\section{Algorithm}

First part of the Algorithm: Discards one of the two features which have a consistent predictable relationship between them in all the instances for all the classes.

\section{Begin}

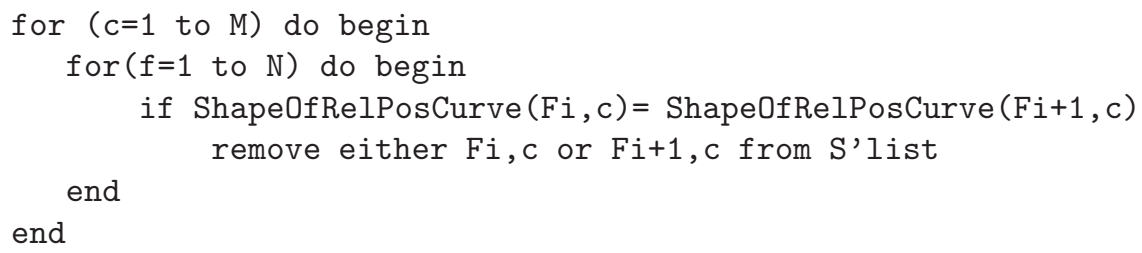

Second part of the Algorithm: Discards the features which are not uniquely related to each of the classes, even if they are very relevant to the classes.

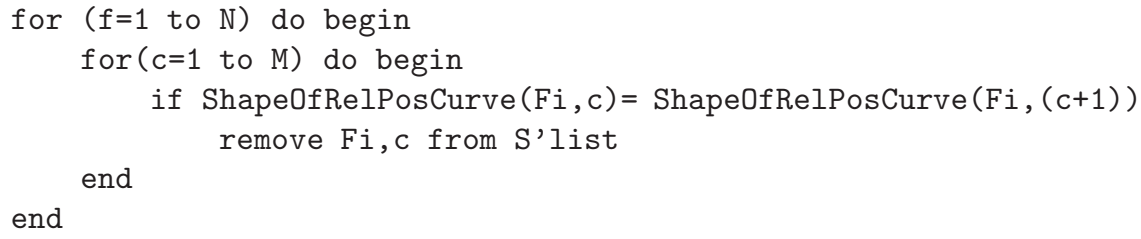


where Fi,c is the $i^{t h}$ feature corresponding to the class c and ShapeOfRelPosCurve(Fi,c) is a function that calculates the width and skew of the curve which contains the relative positions of the input values, corresponding to the feature, $i$ and class, c. This is done by taking percentiles of different points at the curve. The number of points to take depends on the input data (the instance values for each feature of every class). The inputs are:

- Classes, $\mathrm{c}(1,2,3, \ldots . \mathrm{M})$ where ' $\mathrm{c}$ ' refers to 'class' and ranges from 1 to $\mathrm{M}$

- Features, $\mathrm{i}(1,2,3, \ldots \mathrm{N})$ where 'i' refers to 'feature' and ranges from 1 to $\mathrm{N}$ $S^{\prime} l i s t\left(F_{1}, F_{2}, \ldots \ldots . F_{N}\right)$ is the (input) un-optimised set of features

The output is an optimal set of features defined as $S_{\text {best }}$.

\section{Classification}

This paper investigates the use of support vector machines (SVMs) to classify Zernike moments (ZM) into phonemes. SVMs are selected due to the ability of SVMs to find a globally optimum decision function to separate the different classes of data. The training of SVMs involved minimizing the empirical error and complexity of the classifier simultaneously. Good generalization performance in SVMs is achieved by asserting bounds on the classification error and the capacity of the classifiers [14. SVMs can be designed to classify linearly and non-linearly separable data. The data are projected to a higher-dimensional Hilbert space through nonlinear mapping during SVM training. The non linear data are linearly separable using kernel functions in the high-dimensional feature space. This paper implements SVM classifiers with kernel function consisting of Gaussian radial basis function.

\section{Experiments}

The experiments were conducted to evaluate the performance of the proposed lip-reading technique. The vocabulary used in the experiments was based on the viseme model established by an international audiovisual object-based video representation standard known as MPEG-4. Visemes are the atomic units of visual movements associated with phonemes (basic units of speech sound). Visemes can be concatenated to form words and sentences, thus providing the flexibility to expand the vocabulary of the system. The pronunciation of different speech sounds (such as $/ \mathrm{p} /$ and $/ \mathrm{b} /$ ) may be associated with identical visible facial movements and hence each viseme may correspond to more than one phoneme. The MPEG-4 viseme model groups English phonemes into 14 visemes.

To evaluate the performance of the approach in a real world environment, video data was recorded using an inexpensive web camera in a typical office environment. This was done towards having a practical voiceless communication system using low resolution video recordings. The camera focused on the mouth region of the speaker and was kept stationary throughout the experiment. 
The following factors were kept the same during the recording of the videos : window size and view angle of the camera, background and illumination. 2800 utterances of ten subjects were recorded and stored as true color (.AVI) video files. Histogram equalization was applied to the images before computing STT to minimize the effects of illumination variations.

One STT was generated for each utterance. An example of STT for fourteen visemes of a participant are shown in Figure 1. 64 Zernike moments (ZM)

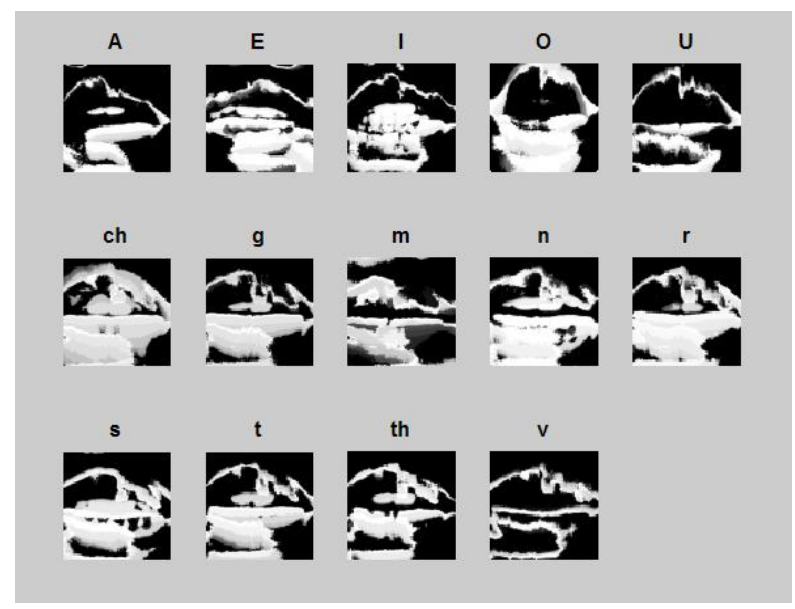

Fig. 1. Example of STT of fourteen visemes

were extracted from each STT. The dimensionality of the features are reduced to 12 values using the proposed feature selection algorithm described in Section 3.1. The reduced features were used to train the SVM classifier. Each viseme was modelled using one SVM. The leave-one-out method was used in the experiment. The average recognition rates of the SVMs for the ten subjects were computed. The SVMs were trained and tested using STT of each individual subjects. The efficiency of the feature reduction technique was tested by comparing the classification accuracies of $12 \mathrm{ZM}$ (after applying feature selection) and the original $64 \mathrm{ZM}$ ( before applying feature selection).

\section{Results}

The classification accuracies of the ten subjects for the 14-phonemes recognition task are tabulated in Table 1. From the results, it is observed that perfect classification accuracy is obtained for each talker (when trained individually) using feature vectors consisting of $12 \mathrm{ZM}$. This suggests that there is no significant intra-subject variation. The high accuracy for all the subjects also indicates that the proposed method for mouth movement representation is not sensitive to skin color and texture. 
Table 1. Average recognition rates for each speaker using ZM features

\begin{tabular}{lcc}
\hline Participants & \multicolumn{2}{c}{ Mean Accuracies (\%) } \\
& 64 ZM & 12 ZM \\
\hline Participant 1 & 99 & 100 \\
Participant 2 & 99 & 100 \\
Participant 3 & 99 & 100 \\
Participant 4 & 98 & 100 \\
Participant 5 & 98 & 100 \\
Participant 6 & 96 & 100 \\
Participant 7 & 96 & 100 \\
Participant 8 & 98 & 100 \\
Participant 9 & 96 & 100 \\
Participant 10 & 98 & 100 \\
\hline
\end{tabular}

The robustness and efficiency of the modified FCBF feature selection algorithm is validated in the experiments. The SVM classification accuracies of $64 \mathrm{ZM}$ features (without using the feature selection algorithm) are reduced by $1 \%$ to $4 \%$ as compared to the $12 \mathrm{ZM}$ features selected using the modified FCBF method. This indicates that the proposed feature reduction algorithm is capable of selecting a much smaller set of optimal features to represent the STT of phonemes.

To compare the results of the proposed approach with other related work is inappropriate due to the different video corpus and recognition tasks used. Researchers who worked on a similar visual-only speech recognition task (based on the the 14 visemes of MPEG-4 standard) has reported approximately $10 \%$ higher error rate using shape-based features (geometric measures of the lip) extracted from static images [15].

\section{Conclusions}

This paper describes a lip-reading approach based on motion segmentation approach and support vector machines. The proposed technique segments mouth movement from image sequences using spatio-temporal templates (STT). Zernike moments (ZM) are computed from STT and used as visual speech features. A new feature selection technique based on fast correlation based filter (FCBF) algorithm is used to reduce the dimensionality of the ZM features. The features are classified using support vector machines (SVM). The results indicate that the proposed technique can produce very high success rates. The results also demonstrate that the proposed feature selection algorithm is capable of reducing the number of features while improving the classification accuracies of SVM. The significant contribution of this paper is the investigation on a novel lipreading technique that combine STT, ZM, modified FCBF-based feature selection method and SVM. Such a system may be applied to drive computerized machinery in noisy environments. The system may also be used for helping disabled people to use a computer. 


\section{References}

1. Petajan, E.D.: Automatic Lip-reading to Enhance Speech Recognition. In: GLOBECOM 1984 (1984)

2. Kaynak, M.N., Qi, Z., Cheok, A.D., Sengupta, K., Chung, K.C.: Audio-visual modeling for bimodal speech recognition. IEEE Transactions on Systems, Man and Cybernetics 34, 564-570 (2001)

3. Potamianos, G., Neti, C., Gravier, G., Garg, A., Senior, A.W.: Recent Advances in Automatic Recognition of Audio-Visual Speech. Proc. of IEEE (2003)

4. Potamianos, G., Neti, C., Huang, J., Connell, J.H., Chu, S., Libal, V., Marcheret, E., Haas, N., Jiang, J.: Towards Practical Deployment of Audio-Visual Speech Recognition. In: ICASSP. IEEE, Los Alamitos (2004)

5. Jourlin, P., Luettin, J., Genoud, D., Wassner, H.: Acoustic-Labial Speaker Verification. In: Bigün, J., Borgefors, G., Chollet, G. (eds.) AVBPA 1997. LNCS, vol. 1206, pp. 319-326. Springer, Heidelberg (1997)

6. Faraj, M., Bigun, J.: Synergy of lip motion and acoustic features in biometric speech and speaker recognition. IEEE trans. Computers 56(9), 1169-1175 (2007)

7. Bobick, A.F., Davis, J.W.: The Recognition of Human Movement Using Temporal Templates. IEEE Transactions on Pattern Analysis and Machine Intelligence 23, 257-267 (2001)

8. Yau, W.C., Kumar, D.K., Arjunan, S.P.: Visual Recognition of Speech Consonants using Facial Movement Features. Integrated Computer-Aided Engineering 14(1), 9-61 (2007)

9. Zhang, D., Lu, G.: Review of Shape Representation and Description Techniques. Pattern Recognition Letters 37 (2004)

10. Teh, C.H., Chin, R.T.: On Image Analysis by the Methods of Moments. IEEE Transactions on Pattern Analysis and Machine Intelligence 10, 496-513 (1988)

11. Khontazad, A., Hong, Y.H.: Invariant Image Recognition by Zernike Moments. IEEE Transactions on Pattern Analysis and Machine Intelligence 12, 489-497 (1990)

12. Yu, L., Liu, H.: Feature Selection for High-Dimensional Data: A Fast CorrelationBased Filter Solution. In: ICML (2003)

13. Cataltepe, Z., Aygun, E., Filiz, A., Keskin, O., Komurlu, C., Altunbasak, Y.: Dimensionality Reduction for Protein Function Prediction. In: Automated Function Prediction(AFP)/ Biosapiens Joint Special Interest Group Meeting, Vienna, Austria (2007)

14. Burges, C.J.C.: A Tutorial on Support Vector Machines for Pattern Recognition. Data Mining and Knowledge Discovery 2(2), 955-974 (1998)

15. Foo, S.W., Dong, L.: Recognition of visual speech elements using hidden Markov models. Lecture notes in computer science. Springer, Heidelberg (2002) 